\title{
Convergence theorems for some multi-valued generalized nonexpansive mappings
}

Shih-sen Chang ${ }^{1 *}$, Yong-kun Tang ${ }^{1}$, Lin Wang ${ }^{1}$, Yu-guang Xu' ${ }^{2}$, Yun-he Zhao ${ }^{1}$ and Gang Wang ${ }^{1}$

Dedicated to Professor W. Takahashi on the occasion of his 70th birthday

${ }^{*}$ Correspondence:

changss2013@aliyun.com

${ }^{1}$ College of Statistics and

Mathematics, Yunnan University of

Finance and Economics, Kunming,

Yunnan 650221, China

Full list of author information is

available at the end of the article

\begin{abstract}
In this paper, we propose an algorithms for finding a common fixed point of an infinite family of multi-valued generalized nonexpansive mappings in uniformly convex Banach spaces. Under suitable conditions some strong and weak convergence theorems for such mappings are proved. The results presented in the paper improve and extend the corresponding results of Suzuki (J. Math. Anal. Appl. 340:1088-1095, 2008), Eslamian and Abkar (Math. Comput. Model. 54:105-111, 2011), Abbas et al. (Appl. Math. Lett. 24:97-102, 2011) and others.

MSC: 47J05; 47H09; 49J25
\end{abstract}

Keywords: multi-valued mapping satisfying condition (C); multi-valued generalized nonexpansive mapping; weak and strong convergence theorem

\section{Introduction}

The study of fixed points for multi-valued contractions and nonexpansive mappings using the Hausdorff metric was initiated by Markin [1] and Nadler [2]. Since then the metric fixed point theory of multi-valued mappings has been rapidly developed. The theory of multi-valued mappings has been applied to control theory, convex optimization, differential equations, and economics. Different iterative processes have been used to approximate fixed points of multi-valued nonexpansive mappings [3-7]. Recently Abbas et al. [8] introduced an one-step iterative process to approximate a common fixed point of two multi-valued nonexpansive mappings in uniformly convex Banach spaces.

On the other hand, in 2008 Suzuki [9] introduced a class of mappings satisfying the condition $(\mathrm{C})$ which is weaker than nonexpansive mappings (sometimes, such a mapping is called a generalized nonexpansive mapping). He then proved some fixed point and convergence theorems for such mappings. Very recently, Eslamian and Abkar $[10,11]$ generalized it to multi-valued case, and they proved some fixed point results in uniformly convex Banach spaces.

The aim of this paper is to introduce an iterative process for approximating a common fixed point of an infinite family of multi-valued mappings satisfying the condition (C). Under suitable conditions some weak and strong convergence theorems for such iterative process are proved in uniformly convex Banach spaces.

o2014 Chang et al.: licensee Springer. This is an Open Access article distributed under the terms of the Creative Commons Attribution License (http://creativecommons.org/licenses/by/2.0), which permits unrestricted use, distribution, and reproduction in any medium, provided the original work is properly cited. 


\section{Preliminaries}

Throughout this paper, we assume that $X$ is a real Banach space, $K$ is a nonempty subset of $X$. We denote by $\mathcal{N}$ the set of all positive integers. We denote by ' $x_{n} \rightarrow x$ ' and ' $x_{n} \rightarrow x$ ' the strong and weak convergence of $\left\{x_{n}\right\}$, respectively.

Recall that a subset $K$ of $X$ is called proximinal if, for each $x \in X$, there exists an element $k^{*} \in K$ such that

$$
d(x, K)=\inf \{\|x-y\|: y \in K\}=d\left(x, k^{*}\right) .
$$

Remark 2.1 It is well-known that weakly compact convex subsets of a Banach space and closed convex subsets of a uniformly convex Banach space are proximinal.

We shall denote the family of nonempty bounded proximinal subsets of $X$ by $P(X)$, the family of nonempty compact subsets of $X$ by $C(X)$ and the family of nonempty bounded and closed subsets of $X$ by $C B(X)$. Let $H$ be the Hausdorff metric induced by the metric $d$ of $X$ defined by

$$
H(A, B):=\max \left\{\sup _{x \in A} d(x, B), \sup _{y \in B} d(y, A)\right\} .
$$

A point $x \in K$ is called a fixed point of a multi-valued mapping $T$, if $x \in T x$. We denote the set of fixed point of $T$ by $F(T)$. A multi-valued mapping $T: K \rightarrow C B(X)$ is said to be

(i) contraction, if there exists a constant $\alpha \in[0,1)$ such that for any $x, y \in K$

$$
H(T x, T y) \leq \alpha\|x-y\|
$$

(ii) nonexpansive, if for all $x, y \in K$

$$
H(T x, T y) \leq\|x-y\|
$$

(iii) quasi-nonexpansive, if $F(T) \neq \emptyset$ and

$$
H(T x, T p) \leq\|x-p\|, \quad \forall p \in F(T), x \in K .
$$

Definition 2.2 A multi-valued mapping $T: X \rightarrow C B(X)$ is said to satisfy the condition (C) provided that

$$
\frac{1}{2} d(x, T x) \leq\|x-y\| \quad \Rightarrow \quad H(T x, T y) \leq\|x-y\|, \quad x, y \in X .
$$

Lemma 2.3 Let $T: X \rightarrow C B(X)$ be a multi-valued mapping.

(1) If $T$ is nonexpansive, then $T$ satisfies the condition (C).

(2) If $T$ satisfies the condition (C) and $F(T) \neq \emptyset$, then $T$ is a quasi-nonexpansive mapping.

Proof The conclusion (1) is obvious. Now we prove the conclusion (2). 
In fact, for any $p \in F(T)$, we have $\frac{1}{2} d(p, T p)=0 \leq\|x-p\|, \forall x \in X$. Since $T$ satisfies the condition $(C)$, we have

$$
H(T x, T p) \leq\|x-p\|, \quad x \in X, p \in F(T) .
$$

We mention that there exist single-valued and multi-valued mappings satisfying the condition $(\mathrm{C})$ which are not nonexpansive, for example:

Example 1 [9] Define a mapping $T$ on [0,3] by

$$
T x= \begin{cases}0, & \text { if } x \neq 3 \\ 1, & \text { if } x=3\end{cases}
$$

Then $T$ is a single-valued mapping satisfying condition (C), but $T$ is not nonexpansive.

Example $2[10]$ Define a mapping $T:[0,5] \rightarrow[0,5]$ by

$$
T(x)= \begin{cases}{\left[0, \frac{x}{5}\right],} & x \neq 5 \\ \{1\}, & x=5\end{cases}
$$

then it is easy to prove that $T$ is a multi-valued mapping satisfying condition (C), but $T$ is not nonexpansive.

Definition 2.4 A Banach space $X$ is said to satisfy Opial condition, if $x_{n} \rightarrow z($ as $n \rightarrow \infty)$ and $z \neq y$ imply that

$$
\limsup _{n \rightarrow \infty}\left\|x_{n}-z\right\|<\limsup _{n \rightarrow \infty}\left\|x_{n}-y\right\|
$$

Lemma 2.5 Let $K$ be a nonempty subset of a uniformly convex Banach space $X$ and $T: K \rightarrow C B(K)$ be a multi-valued mapping with convex-valued and satisfying the condition $(C)$, then

$$
H(T x, T y) \leq 2 d(x, T x)+\|x-y\|, \quad \forall x, y \in K .
$$

Proof Let $x \in K$, since $T x$ is a nonempty closed and convex subset of $K$. By Remark 2.1, it is proximal, hence there exists $z \in T x$ such that $\|z-x\|=d(x, T x)$. Since $\frac{1}{2} d(x, T x) \leq\|z-x\|$ and $T$ satisfies the condition (C), we have

$$
H(T x, T z) \leq\|z-x\| .
$$

Also since $T z$ is proximal, there exists $u \in T z$ such that $\|z-u\|=d(z, T z)$. This together with (2.1) shows that

$$
\|z-u\|=d(z, T z) \leq H(T x, T z) \leq\|z-x\| .
$$

Now we show that either $\frac{1}{2} d(x, T x) \leq\|x-y\|$ or $\frac{1}{2} d(z, T z) \leq\|z-y\|$ holds. Suppose to the contrary, we may assume that

$$
\frac{1}{2} d(x, T x)>\|x-y\| \quad \text { and } \quad \frac{1}{2} d(z, T z)>\|z-y\| .
$$


From (2.2) we have

$$
\begin{aligned}
\|z-x\| & \leq\|x-y\|+\|y-z\|<\frac{1}{2} d(x, T x)+\frac{1}{2} d(z, T z) \\
& \leq \frac{1}{2}\|z-x\|+\frac{1}{2}\|z-x\|=\|z-x\|
\end{aligned}
$$

which is a contradiction. If $\frac{1}{2} d(x, T x) \leq\|x-y\|$, then by the fact that $T$ satisfies the condition $(C)$, we have $H(T x, T y) \leq\|x-y\|$. In the other case, if $\frac{1}{2} d(z, T z) \leq\|z-y\|$, again by the assumption that $T$ satisfies the condition (C) we obtain $H(T z, T y) \leq\|z-y\|$. Hence, we get

$$
\begin{aligned}
H(T x, T y) & \leq H(T x, T z)+H(T z, T y) \leq\|z-x\|+\|z-y\| \\
& \leq\|z-x\|+\|z-x\|+\|x-y\| \\
& =2 d(x, T x)+\|x-y\|, \quad x, y \in K .
\end{aligned}
$$

This completes the proof of Lemma 2.5.

Lemma 2.6 [12] Let $X$ be a uniformly convex Banach space, $B_{r}(0):=\{x \in X:\|x\| \leq r\}$ be a closed ball with center 0 and radius $r>0$. For any given sequence $\left\{x_{1}, x_{2}, \ldots, x_{n}, \ldots\right\} \subset B_{r}(0)$ and any given number sequence $\left\{\lambda_{1}, \lambda_{2}, \ldots, \lambda_{n}, \ldots\right\}$ with $\lambda_{i} \geq 0, \sum_{i=1}^{\infty} \lambda_{i}=1$, then there exists a continuous strictly increasing and convex function $g:[0,2 r) \rightarrow[0, \infty)$ with $g(0)=0$ such that for any $i, j \in \mathcal{N}, i<j$ the following holds:

$$
\left\|\sum_{n=1}^{\infty} \lambda_{n} x_{n}\right\|^{2} \leq \sum_{n=1}^{\infty} \lambda_{n}\left\|x_{n}\right\|^{2}-\lambda_{i} \lambda_{j} g\left(\left\|x_{i}-x_{j}\right\|\right) .
$$

Lemma 2.7 Let $X$ be a strictly convex Banach space, $K$ be a nonempty closed and convex subset of $X$ and $T: K \rightarrow C B(K)$ be a multi-valued mapping satisfying the condition $(C)$. If $F(T)$ is nonempty, then it is a closed and convex subset of $K$.

Proof Let $\left\{x_{n}\right\}$ be a sequence in $F(T)$ converging to some point $p \in K$, i.e., $x_{n} \in T\left(x_{n}\right)$, $\forall n \geq 1$ and $x_{n} \rightarrow p \in K$. Since $T$ satisfies the condition $(C)$ and

$$
\frac{1}{2} d\left(x_{n}, T x_{n}\right)=0 \leq\left\|x_{n}-p\right\|, \quad \forall n \geq 1,
$$

we have

$$
d(p, T p)=\limsup _{n \rightarrow \infty} d\left(x_{n}, T p\right) \leq \limsup _{n \rightarrow \infty} H\left(T x_{n}, T p\right) \leq \limsup _{n \rightarrow \infty}\left\|x_{n}-p\right\|=0
$$

This implies that $d(p, T p)=0$. Since $T p$ is closed, we have $p \in T p$, i.e., $p \in F(T)$ and so $F(T)$ is closed.

Next we prove that $F(T)$ is a convex subset in $K$. In fact, for any given $\lambda \in(0,1), x, y \in$ $F(T)$ with $x \neq y$ and put $w=\lambda x+(1-\lambda) y$. Since $T$ satisfies the condition $(C)$, we have

$$
\begin{aligned}
\|x-y\| & \leq d(x, T w)+d(y, T w) \leq H(T x, T w)+H(T y, T w) \\
& \leq\|x-w\|+\|y-w\|=(1-\lambda)\|x-y\|+\lambda\|x-y\|=\|x-y\| .
\end{aligned}
$$


This implies that

$$
\|x-y\|=d(x, T w)+d(y, T w)=\|x-w\|+\|y-w\|=\|x-y\| .
$$

Since $X$ is strictly convex, this implies that there exist $\mu \in(0,1)$ and a point $u \in T w$ such that $u=\mu x+(1-\mu) y$. Since

$$
\begin{aligned}
(1-\mu)\|x-y\| & =\|x-u\| \leq d(x, T x)+H(T x, T w)+d(T w, u) \\
& \leq\|x-w\|=(1-\lambda)\|x-y\|
\end{aligned}
$$

and

$$
\begin{aligned}
\mu\|x-y\| & =\|y-u\| \leq d(y, T y)+H(T y, T w)+d(T w, u) \\
& \leq\|y-w\|=\lambda\|x-y\|,
\end{aligned}
$$

from (2.4), we have $1-\mu \leq(1-\lambda)$ and from (2.5), we have $\mu \leq \lambda$. These implies that $\mu=\lambda$. Therefore $u=w$ and $w \in T w$, i.e., $w \in F(T)$. This completes the proof of Lemma 2.7.

Lemma 2.8 (Demi-closed principle) Let $X$ be a uniformly convex Banach space satisfying the Opial condition, $K$ be a nonempty closed and convex subset of $X$. Let $T: K \rightarrow C B(K)$ be a multi-valued mapping with convex-values and satisfying the condition $(C)$. Let $\left\{x_{n}\right\}$ be a sequence in $K$ such that $x_{n} \rightarrow p \in K$, and let $\lim _{n \rightarrow \infty} d\left(x_{n}, T x_{n}\right)=0$, then $p \in T p$, i.e., $I-T$ is demi-closed at zero.

Proof By the assumption that $T: K \rightarrow C B(K)$ is a multi-valued mapping with convexvalues, hence $T p$ is a nonempty closed and convex subset of $K$. By Remark 2.1, it is proximal. Therefore for each $x_{n}, n \geq 1$, there exists a point $u_{n} \in T p$ such that

$$
\left\|x_{n}-u_{n}\right\|=d\left(x_{n}, T p\right), \quad n \geq 1
$$

On the other hand, it follows from Lemma 2.5 that

$$
\begin{aligned}
\left\|x_{n}-u_{n}\right\| & =d\left(x_{n}, T p\right) \leq d\left(x_{n}, T x_{n}\right)+H\left(T x_{n}, T p\right) \\
& \leq d\left(x_{n}, T x_{n}\right)+2 d\left(x_{n}, T x_{n}\right)+\left\|x_{n}-p\right\|, \quad \forall n \geq 1 .
\end{aligned}
$$

Taking the superior limit on the both sides of the above inequality, we have

$$
\limsup _{n \rightarrow \infty}\left\|x_{n}-u_{n}\right\| \leq \limsup _{n \rightarrow \infty}\left\|x_{n}-p\right\| .
$$

By virtue of the Opial condition, we have $u_{n}=p, \forall n \geq 1$. And so $p \in T p$.

This completes the proof of Lemma 2.8 .

\section{Weak convergence theorems}

We are now in a position to give the following theorem. 
Theorem 3.1 Let $X$ be a real uniformly convex Banach space with Opial condition and $K$ be a nonempty closed and convex subset of $X$. Let $T_{i}: K \rightarrow C B(K), i=1,2, \ldots$ be an infinite family of multi-valued mappings with nonempty convex-values and satisfying the condition (C). For given $x_{1} \in K$, let $\left\{x_{n}\right\}$ be the sequence in $K$ defined by

$$
x_{n+1}=\alpha_{0, n} x_{n}+\sum_{i=1}^{\infty} \alpha_{i, n} w_{i, n}, \quad w_{i, n} \in T_{i} x_{n}, n \geq 1,
$$

where $\left\{\alpha_{i, n}\right\} \subset(0,1)$. If the following conditions are satisfied:

(i) $\sum_{i=0}^{\infty} \alpha_{i, n}=1$, for each $n \geq 1$;

(ii) for each $i \geq 1$, $\liminf _{n \rightarrow \infty} \alpha_{0, n} \alpha_{i, n}>0$;

(iii) $\mathcal{F}:=\bigcap_{i=1}^{\infty} F\left(T_{i}\right) \neq \emptyset$ and $T_{i} p=\{p\}, \forall i \geq 1$ and $p \in \mathcal{F}$,

then the sequence $\left\{x_{n}\right\}$ converges weakly to some point $p^{*} \in \mathcal{F}$.

Proof (I) First we claim that

$$
\lim _{n \rightarrow \infty}\left\|x_{n}-p\right\| \text { exists for each } p \in \mathcal{F} .
$$

In fact, since $\bigcap_{i=1}^{\infty} F\left(T_{i}\right) \neq \emptyset$, it follows from Lemma 2.3(2) that for each $i \geq 1, T_{i}$ is a multi-valued quasi-nonexpansive mapping. Hence for each $p \in \mathcal{F}$, by condition (iii) we have

$$
\left.\left\|w_{i, n}-p\right\|=d\left(w_{i, n}, T_{i} p\right) \leq H\left(T_{i} x_{n}\right), T_{i} p\right) \leq\left\|x_{n}-p\right\|, \quad \forall n \geq 1
$$

and

$$
\begin{aligned}
\left\|x_{n+1}-p\right\| & =\left\|\alpha_{0, n} x_{n}+\sum_{i=1}^{\infty} \alpha_{i, n} w_{i, n}-p\right\| \leq \alpha_{0, n}\left\|x_{n}-p\right\|+\sum_{i=1}^{\infty} \alpha_{i, n}\left\|w_{i, n}-p\right\| \\
& \leq \alpha_{0, n}\left\|x_{n}-p\right\|+\sum_{i=1}^{\infty} \alpha_{i, n}\left\|x_{n}-p\right\| \\
& =\left\|x_{n}-p\right\|, \quad \forall n \geq 1 .
\end{aligned}
$$

This shows that $\left\{\left\|x_{n}-p\right\|\right\}$ is decreasing and bounded below. Hence the $\operatorname{limit} \lim _{n \rightarrow \infty} \| x_{n}-$ $p \|$ exists for each $p \in \mathcal{F}$. And so $\left\{\left\|x_{n}-p\right\|\right\}$ and $\left\{\left\|w_{i, n}-p\right\|\right\}$ both are bounded.

(II) Next we prove that

$$
\lim _{n \rightarrow \infty} d\left(x_{n}, T_{l} x_{n}\right)=0 \quad \text { for each } l \geq 1 .
$$

Since $\left\{\left\|x_{n}-p\right\|\right\}$ and $\left\{\left\|w_{i, n}-p\right\|\right\}$ both are bounded, from Lemma 2.6 and (3.3), for each $l \geq 1$ we have

$$
\begin{aligned}
\left\|x_{n+1}-p\right\|^{2} & =\left\|\alpha_{0, n}\left(x_{n}-p\right)+\sum_{i=1}^{\infty} \alpha_{i, n}\left(w_{i, n}-p\right)\right\|^{2} \\
& \leq \alpha_{0, n}\left\|x_{n}-p\right\|^{2}+\sum_{i=1}^{\infty} \alpha_{i, n}\left\|w_{i, n}-p\right\|^{2}-\alpha_{0, n} \alpha_{l, n} g\left(\left\|x_{n}-w_{l, n}\right\|\right)
\end{aligned}
$$




$$
\begin{aligned}
& \leq \alpha_{0, n}\left\|x_{n}-p\right\|^{2}+\sum_{i=1}^{\infty} \alpha_{i, n}\left\|x_{n}-p\right\|^{2}-\alpha_{0, n} \alpha_{l, n} g\left(\left\|x_{n}-w_{l, n}\right\|\right) \\
& =\left\|x_{n}-p\right\|^{2}-\alpha_{0, n} \alpha_{l, n} g\left(\left\|x_{n}-w_{l, n}\right\|\right), \quad \forall n \geq 1 .
\end{aligned}
$$

And so

$$
\alpha_{0, n} \alpha_{l, n} g\left(\left\|x_{n}-w_{l, n}\right\|\right) \leq\left\|x_{n}-p\right\|^{2}-\left\|x_{n+1}-p\right\|^{2} \rightarrow 0 \quad(\text { as } n \rightarrow \infty) .
$$

By condition (ii) we have

$$
\lim _{n \rightarrow \infty} g\left(\left\|x_{n}-w_{l, n}\right\|\right)=0
$$

Since $g$ is continuous and strictly increasing with $g(0)=0$, this implies that

$$
\lim _{n \rightarrow \infty}\left\|x_{n}-w_{l, n}\right\|=0, \quad \forall l \geq 1
$$

Thus, we have

$$
\lim _{n \rightarrow \infty} d\left(x_{n}, T_{l} x_{n}\right) \leq \lim _{n \rightarrow \infty}\left\|x_{n}-w_{l, n}\right\|=0, \quad \forall l \geq 1
$$

(III) Finally we prove that $x_{n} \rightarrow p^{*}$ (some point in $\mathcal{F}$ ).

In fact, since $\left\{x_{n}\right\}$ is bounded, there exists a subsequence $x_{n_{i}} \subset\left\{x_{n}\right\}$ such that $x_{n_{i}} \rightarrow$ $p^{*} \in K$. By Lemma 2.8, $I-T_{l}$ is demi-closed at zero. Hence from (3.8), $p^{*} \in F\left(T_{l}\right)$. By the arbitrariness of $l \geq 1$, we have $p^{*} \in \mathcal{F}$.

If there exists another subsequence $\left\{x_{n_{j}}\right\} \subset\left\{x_{n}\right\}$ such that $x_{n_{j}} \rightarrow q^{*} \in K$ and $p^{*} \neq q^{*}$. By the same method as given above we can also prove that $q^{*} \in \mathcal{F}$. Since $X$ has the Opial property, we have

$$
\begin{aligned}
\limsup _{n_{i} \rightarrow \infty}\left\|x_{n_{i}}-p^{*}\right\| & <\limsup _{n_{i} \rightarrow \infty}\left\|x_{n_{i}}-q^{*}\right\|=\lim _{n \rightarrow \infty}\left\|x_{n}-q^{*}\right\| \\
& =\limsup _{n_{j} \rightarrow \infty}\left\|x_{n_{j}}-q^{*}\right\|<\underset{n_{j} \rightarrow \infty}{\limsup }\left\|x_{n_{j}}-p^{*}\right\| \\
& =\lim _{n \rightarrow \infty}\left\|x_{n}-p^{*}\right\|=\limsup _{n_{i} \rightarrow \infty}\left\|x_{n_{i}}-p^{*}\right\| .
\end{aligned}
$$

This is a contradiction. Therefore $p^{*}=q^{*}$ and $x_{n} \rightarrow p^{*} \in \mathcal{F}$.

This completes the proof of Theorem 3.1.

The following theorem can be obtained from Theorem 3.1 immediately.

Theorem 3.2 Let $X$ be a real uniformly convex Banach space with Opial condition and $K$ be a nonempty closed and convex subset of $X$. Let $T_{i}: K \rightarrow K, i=1,2, \ldots$ be an infinite family of single-valued mappings satisfying the condition $(\mathrm{C})$. For given $x_{1} \in K$, let $\left\{x_{n}\right\}$ be the sequence in $K$ defined by

$$
x_{n+1}=\alpha_{0, n} x_{n}+\sum_{i=1}^{\infty} \alpha_{i, n} T_{i} x_{n}, \quad n \geq 1,
$$


where $\left\{\alpha_{i, n}\right\} \subset(0,1)$ is the sequence as given in Theorem 3.1. If $\mathcal{F}:=\bigcap_{i=1}^{\infty} F\left(T_{i}\right) \neq \emptyset$, then the sequence $\left\{x_{n}\right\}$ converges weakly to some point $p^{*} \in \mathcal{F}$.

Remark 3.3 Theorem 3.1 improves and extends the corresponding results in Eslamian and Abkar [11, Theorem 3.3], Suzuki [9, Theorem 3] and Abbas et al. [8, Theorem 2].

\section{Some strong convergence theorems}

Theorem 4.1 Let $X$ be a real uniformly convex Banach space and $K$ be a nonempty closed and convex subset of $X$. Let $T_{i}: K \rightarrow C B(K), i=1,2, \ldots$ be an infinite family of multi-valued mappings satisfying the condition $(C)$. For given $x_{1} \in K$, let $\left\{x_{n}\right\}$ be the sequence defined by (3.1). If the conditions (i), (ii), and (iii) in Theorem 3.1 are satisfied, then $\left\{x_{n}\right\}$ converges strongly to some point $p^{*} \in \mathcal{F}$, if and only if the following condition is satisfied:

$$
\liminf _{n \rightarrow \infty} d\left(x_{n}, \mathcal{F}\right)=0
$$

Proof The necessity of condition (4.1) is obvious.

Next we prove the sufficiency of condition (4.1).

In fact, as in the proof of Theorem 3.1, for each $i \geq 1$, we have $\lim d\left(x_{n}, T_{i} x_{n}\right)=0$ (see (3.8)), and for each $p \in \mathcal{F}$ the $\operatorname{limit}_{n \rightarrow \infty}\left\|x_{n}-p\right\|$ exists. Hence by condition (4.1) we have

$$
\lim _{n \rightarrow \infty} d\left(x_{n}, \mathcal{F}\right)=0
$$

Therefore we can choose a subsequence $\left\{x_{n_{k}}\right\} \subset\left\{x_{n}\right\}$ and a subsequence $\left\{p_{k}\right\} \subset \mathcal{F}$ such that for all positive integer $k \geq 1$

$$
\left\|x_{n_{k}}-p_{k}\right\|<\frac{1}{2^{k}}
$$

Since the sequence $\left\{\left\|x_{n}-p\right\|\right\}, p \in \mathcal{F}$ is decreasing, we obtain

$$
\left\|x_{n_{k+1}}-p_{k}\right\| \leq\left\|x_{n_{k}}-p_{k}\right\|<\frac{1}{2^{k}}
$$

Hence

$$
\left\|p_{n_{k+1}}-p_{k}\right\| \leq\left\|x_{n_{k+1}}-p_{k+1}\right\|+\left\|x_{n_{k+1}}-p_{k}\right\|<\frac{1}{2^{k+1}}+\frac{1}{2^{k}}<\frac{1}{2^{k-1}} .
$$

This implies that $\left\{p_{k}\right\}$ is a Cauchy sequence in $K$. Without loss of generality, we can assume that $p_{k} \rightarrow p^{*} \in K$. Since for each $i \geq 1$

$$
d\left(p^{*}, T_{i}\left(p^{*}\right)\right)=\lim _{k \rightarrow \infty} d\left(p_{k}, T_{i}\left(p^{*}\right)\right) \leq \lim _{k \rightarrow \infty} H\left(T_{i}\left(p_{k}\right), T_{i}\left(p^{*}\right)\right) \leq \lim _{k \rightarrow \infty}\left\|p_{k}-p^{*}\right\|=0
$$

This implies that $p^{*} \in T_{i} p^{*}$, for all $i \geq 1$. Therefore $p^{*} \in \mathcal{F}$ and $x_{n} \rightarrow p^{*}$.

This completes the proof of Theorem 4.1.

The following theorem can be obtained from Theorem 4.1 immediately. 
Theorem 4.2 Let $X$ be a real uniformly convex Banach space and $K$ be a nonempty closed and convex subset of $X$. Let $T_{i}: K \rightarrow C B(K), i=1,2, \ldots$ be an infinite family of multi-valued mappings satisfying the condition (C). For given $x_{1} \in K$, let $\left\{x_{n}\right\}$ be the sequence defined by (3.1). If the conditions (i), (ii), and (iii) in Theorem 3.1 and the following condition (iv) are satisfied:

(iv) there exists an increasing function $f:[0, \infty) \rightarrow[0, \infty)$ with $f(r)>0, \forall r>0$ such that for some $m \geq 1$

$$
d\left(x_{n}, T_{m}\left(x_{n}\right)\right) \geq f\left(d\left(x_{n}, \mathcal{F}\right)\right),
$$

then the sequence $\left\{x_{n}\right\}$ converges strongly to some point $p^{*} \in \mathcal{F}$.

Proof As in the proof of Theorem 3.1, for each $i \geq 1, \lim _{n \rightarrow \infty} d\left(x_{n}, T_{i} x_{n}\right)=0$. Especially we have $\lim _{n \rightarrow \infty} d\left(x_{n}, T_{m} x_{n}\right)=0$. Hence from (4.3) we obtain $\lim _{n \rightarrow \infty} d\left(x_{n}, \mathcal{F}\right)=0$. The conclusion of Theorem 4.2 can be obtained from Theorem 4.1 immediately.

We now intend to remove the condition that $T_{i}(p)=\{p\}$ for each $p \in \mathcal{F}$ and each $i \geq 1$.

Let $X$ be a real uniformly convex Banach space and $K$ be a nonempty closed and convex subset of $X$. Let $T_{i}: K \rightarrow C B(K), i=1,2, \ldots$ be an infinite family of multi-valued mappings with convex-values. Then for each $i \geq 1$ and for each $x \in K, T_{i} x$ is a nonempty closed and convex subset in $K$. Hence by Remark 2.1, it is proximinal. Now we define a multi-valued mapping $P_{T_{i}}: K \rightarrow C B(K)$ by

$$
P_{T_{i}}(x)=\left\{y \in T_{i}(x):\|x-y\|=d\left(x, T_{i}(x)\right)\right\} .
$$

For any given $x_{1} \in K$ define a sequence $\left\{x_{n}\right\}$ by

$$
x_{n+1}=\alpha_{0, n} x_{n}+\sum_{i=1}^{\infty} \alpha_{i, n} w_{i, n}, \quad w_{i, n} \in P_{T_{i}}\left(x_{n}\right), n \geq 1
$$

where $\left\{\alpha_{i, n}\right\} \subset(0,1)$.

We have the following.

Theorem 4.3 Let $X, K,\left\{T_{i}\right\},\left\{P_{T_{i}}\right\}$ and $\left\{x_{n}\right\}$ be the same as above. If the following conditions are satisfied:

(i) $\sum_{i=0}^{\infty} \alpha_{i, n}=1$, for each $n \geq 1$;

(ii) for each $i \geq 1$, $\liminf _{n \rightarrow \infty} \alpha_{0, n} \alpha_{i, n}>0$;

(iii) $\mathcal{F}:=\bigcap_{i=1}^{\infty} F\left(T_{i}\right) \neq \emptyset$, and, for each $i \geq 1$, the mapping $P_{T_{i}}: K \rightarrow C B(K)$ satisfies the condition (C);

(iv) there exists an increasing function $f:[0, \infty) \rightarrow[0, \infty)$ with $f(r)>0$ for all $r>0$ such that for some $m \geq 1$

$$
d\left(x_{n}, T_{m}\left(x_{n}\right)\right) \geq f\left(d\left(x_{n}, \mathcal{F}\right)\right),
$$

then the sequence $\left\{x_{n}\right\}$ converges strongly to some point $p^{*} \in \mathcal{F}$. 
Proof Let $p \in \mathcal{F}$, then we have

$$
P_{T_{i}}(p)=\left\{y \in T_{i}(p):\|p-y\|=d\left(p, T_{i}(p)\right)=0\right\}=\{p\} \quad \text { for each } i \geq 1 .
$$

Moreover, by the same method as give in the proof of Theorem 3.1 we can also prove that the limit $\lim _{n \rightarrow \infty}\left\|x_{n}-p\right\|$ exists for each $p \in \mathcal{F}$ and

$$
\lim _{n \rightarrow \infty} d\left(x_{n}, \mathcal{F}\right)=0
$$

Therefore, we can choose a subsequence $\left\{x_{n_{k}}\right\}$ of $\left\{x_{n}\right\}$ and a sequence $\left\{p_{k}\right\}$ in $\mathcal{F}$ such that for any positive integer $k$

$$
\left\|x_{n_{k}}-p_{k}\right\|<\frac{1}{2^{k}}
$$

As in the proof of Theorem 3.1, $\left\{p_{k}\right\}$ is a Cauchy sequence in $K$ and hence it converges to some point $q \in K$. By virtue of the definition of mapping $P_{T_{i}}$, we have $P_{T_{i}}(q) \subset T_{i}(q), i \geq 1$. Hence from (4.6) we have

$$
d\left(p_{k}, T_{i}(q)\right) \leq d\left(p_{k}, P_{T_{i}}(q)\right) \leq H\left(P_{T_{i}}\left(p_{k}\right), P_{T_{i}}(q)\right) \leq\left\|q-p_{k}\right\| .
$$

Since $p_{k} \rightarrow q($ as $k \rightarrow \infty)$, it follows that $d\left(q, T_{i}(q)\right)=0$ for $i \geq 1$. Hence $q \in \mathcal{F}$ and $\left\{x_{n_{k}}\right\}$ converges strongly to $q$. Since $\lim _{n \rightarrow \infty}\left\|x_{n}-q\right\|$ exists, we conclude that $\left\{x_{n}\right\}$ converges strongly to $q$.

This completes the proof of Theorem 4.3.

Remark 4.4 Theorems 4.1, 4.2 and 4.3 improve and extend the corresponding results in Eslamian et al. [11, Theorems 3.1, 3.2, 3.4], Suzuki [9, Theorem 2] and Abbas et al. [8, Theorems 1, 3, 4].

\section{Applications}

The convex feasibility problem (CFP) was first introduced by Censor and Elfving [13] for modeling inverse problems which arise from phase retrievals and in medical image reconstruction [14]. Recently, it has been found that the CFP can also be used in various disciplines such as image restoration, computer tomograph and radiation therapy treatment planning.

Let $X$ be a real Banach space, $K$ be a nonempty closed and convex subset of $X$ and $\left\{K_{i}\right\}$ be a countable family of subset of $K$. The 'so called' convex feasibility problem for the family of subsets $\left\{K_{i}\right\}$ is to find a point $x^{*} \in \bigcap_{i=1}^{\infty} K_{i}$.

In this section, we shall utilize Theorem 3.2 to study the convex feasibility problem for an infinite family of single-valued mappings satisfying the condition (C). We have the following result.

Theorem 5.1 Let $X$ be a real uniformly convex Banach space with Opial condition and $K$ be a nonempty closed and convex subset of $X$. Let $T_{i}: K \rightarrow K, i=1,2, \ldots$ be an infinite family of mappings satisfying the condition (C). Let $\left\{K_{i}=F\left(T_{i}\right), i=1,2, \ldots\right\}$. For given $x_{1} \in K$, let 
$\left\{x_{n}\right\}$ be the sequence in $K$ defined by

$$
x_{n+1}=\alpha_{0, n} x_{n}+\sum_{i=1}^{\infty} \alpha_{i, n} T_{i} x_{n}, \quad n \geq 1,
$$

where $\left\{\alpha_{i, n}\right\} \subset(0,1)$ is the sequence as given in Theorem 3.2. If $\mathcal{F}:=\bigcap_{i=1}^{\infty} F\left(T_{i}\right) \neq \emptyset$, then there exists a point $x^{*} \in \mathcal{F}$ which is a solution of the convex feasibility problem for the family of subsets $\left\{K_{i}\right\}$, and the sequence $\left\{x_{n}\right\}$ defined by (5.1) converges weakly to $x^{*}$.

\section{Competing interests}

The authors declare that they have no competing interests.

\section{Authors' contributions}

All authors contributed equally and significantly to this research work. All authors read and approved the final manuscript.

\section{Author details}

${ }^{1}$ College of Statistics and Mathematics, Yunnan University of Finance and Economics, Kunming, Yunnan 650221, China.

${ }^{2}$ Department of Mathematics, Kunming University, Kunming, Yunnan 650214, China.

\section{Acknowledgements}

The authors would like to express their thanks to the Reviewers and the Editors for their helpful suggestions and advices. This work was supported by the National Natural Science Foundation of China (Grant No. 11361070).

Received: 7 September 2013 Accepted: 24 January 2014 Published: 11 Feb 2014

\section{References}

1. Markin, J: A fixed point theorem for set valued mappings. Bull. Am. Math. Soc. 74, 639-640 (1968)

2. Nadler, SB Jr.: Multi-valued contraction mappings. Pac. J. Math. 30, 475-488 (1969)

3. Sastry, KPR, Babu, GVR: Convergence of Ishikawa iterates for a multivalued mapping with a fixed point. Czechoslov. Math. J. 55, 817-826 (2005)

4. Panyanak, B: Mann and Ishikawa iterative processes for multivalued mappings in Banach spaces. Comput. Math. Appl. 54, 872-877 (2007)

5. Song, Y, Wang, H: Erratum to Mann and Ishikawa iterative processes for multivalued mappings in Banach spaces. Comput. Math. Appl. 55, 2999-3002 (2008)

6. Song, $\mathrm{Y}, \mathrm{Wang}, \mathrm{H}$ : Convergence of iterative algorithms for multivalued mappings in Banach spaces. Nonlinear Anal. 70, 1547-1556 (2009)

7. Shahzad, N, Zegeye, $\mathrm{H}$ : On Mann and Ishikawa iteration schemes for multivalued maps in Banach space. Nonlinear Anal. 71, 838-844 (2009)

8. Abbas, M, Khan, SH, Khan, AR, Agarwal, RP: Common fixed points of two multivalued nonexpansive mappings by one-step iterative scheme. Appl. Math. Lett. 24, 97-102 (2011)

9. Suzuki, T: Fixed point theorems and convergence theorems for some generalized nonexpansive mappings. J. Math. Anal. Appl. 340, 1088-1095 (2008)

10. Abkar, A, Eslamian, M: Fixed point theorems for Suzuki generalized nonexpansive multivalued mappings in Banach spaces. Fixed Point Theory Appl. 2010, Article ID 457935 (2010)

11. Eslamian, M, Abkar, A: One-step iterative process for a finite family of multivalued mappings. Math. Comput. Model. 54, 105-111 (2011)

12. Chang, SS, Kim, JK, Wang, XR: Modified block iterative algorithm for solving convex feasibility problems in Banach spaces. J. Inequal. Appl. 2010, Article ID 869684 (2010). doi:10.1155/2010/869684

13. Censor, Y, Elfving, T: A multi-projection algorithm using Bregman projections in a product space. Numer. Algorithms 8, 221-239 (1994)

14. Byrne, C: Iterative oblique projection onto convex subsets and the split feasibility problem. Inverse Probl. 18, 441-453 (2002) 